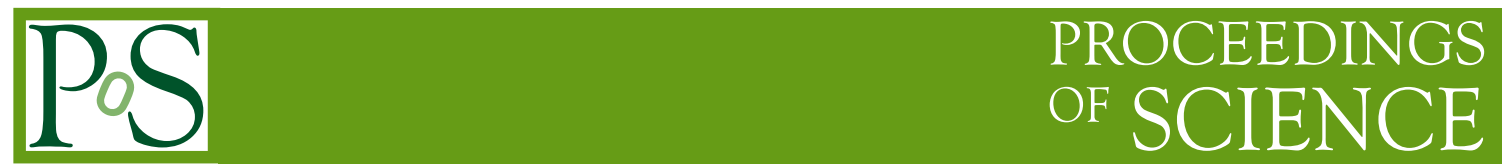

\title{
Lecture Notes on D-brane Models with Fluxes
}

\section{Ralph Blumenhagen*}

Max-Planck-Institut für Physik, Föhringer Ring 6, D-80805 München, GERMANY

E-mail: blumenha@mppmu.mpg.de

This is a set of lecture notes for the RTN Winter School on Strings, Supergravity and Gauge Theories, January 31-February 4 2005, SISSA/ISAS Trieste. On a basic (inevitably sometimes superficial) level, intended to be suitable for students, recent developments in string model building are covered. This includes intersecting D-brane models, flux compactifications, the KKLT scenario and the statistical approach to the string vacuum problem.

RTN Winter School on Strings, Supergravity and Gauge Theories

$31 / 1-4 / 22005$

SISSA, Trieste, Italy

${ }^{*}$ Speaker. 


\section{Contents}

1. D-brane models 2

1.1 Gauge fields on D-branes

1.2 Orientifolds

1.3 Chirality 6

1.4 T-duality 7

1.5 Intersecting D-brane models 7

2. Flux compactifications 9

2.1 New tadpoles 10

2.2 The scalar potential 11

3. The KKLT scenario 12

3.1 AdS minima 13

3.2 dS minima 13

4. Toward realistic brane and flux compactifications

4.1 A supersymmetric $G_{3}$ flux 15

4.2 MSSM like magnetized D-branes 17

5. Statistics of string vacua 18

\section{D-brane models}

It is still a great challenge for string theory to answer the basic question whether it has anything to do with nature. In the framework of string theory this question boils down to whether string theory incorporates the Standard Model (SM) of particle physics at low energies or whether string theory has a vacuum/vacua resembling our world to the degree of accuracy with which it has been measured. This latter formulation implies that neither do we know that string theory contains at least one such vacuum nor do we know, if there exists a solution, whether it is unique.

Clearly, in order to make progress we have to know what Standard Model like vacua string theory admits. For an admissible string vacuum we at least have to ensure that the following "first order requirements" are satisfied.

- Gauge symmetry $S U(3)_{c} \times S U(2)_{w} \times U(1)_{Y}$

- Three generations of chiral SM fermions, including right handed neutrinos

- Mechanism to break $S U(2)_{w} \times U(1)_{Y} \rightarrow U(1)_{e m}$, Higgs particles 
- Mechanism to stabilize the weak scale: supersymmetry

We will see that these properties can be fairly easily controlled in string theory and are essentially given by topological features of the string background. However, having a candidate vacuum satisfying all these constraints there are additional "second order requirements".

- Yukawa couplings $\rightarrow$ fermion masses, $C P$ violation

- Details of supersymmetry breaking, concrete form of soft terms, suppression of FCNCs

- Gauge couplings resp. gauge coupling unification

- Cosmological issues: smallness of the cosmological constant, inflationary scenario, etc.

In this lecture we will get to know a concrete class of string models which does provide mechanisms to satisfy at least the first order requirements. These models use intersecting/magnetized D-branes as their essential ingredient. For recent reviews on this subject please consult [1, 2, 3, 4. 6, 6, 7, 8]. [Since these are lecture notes, I will not try to provide a complete list of references. Instead I will mainly refer to those papers, whose content is directly covered in these lecture notes. Any overlap with our recent review articles could hardly be avoided and is intended. Therefore, I apologize to those readers who meanwhile feel bored by seeing the same topic presented by the same people ad nauseam.]

\subsection{Gauge fields on D-branes}

First one needs a mechanism to get gauge symmetries in string theory. One natural source are the lightest open string excitations, where in general the ends of the open strings can end on D-branes. More concretely, a p-brane is an extended object with $\mathrm{p}$ space-like directions and one time-like direction and it couples to a $(p+1)$ form potential $C_{p+1}$ as follows:

$$
S_{p}=Q_{p} \int_{D_{p}} C_{p+1},
$$

where the integral is over the $(p+1)$ dimensional world-volume of the D-brane and $Q_{p}$ denotes its $\mathrm{R}-\mathrm{R}$ charge. For BPS D-branes in Type IIA string theory $p$ is an even number and in Type IIB an odd one. Polchinski was the first to realize that the fluctuations of such D-branes can by themselves be described by a string theory [9](see [10, 11] for reviews), which in this case are open strings attached to the D-brane, i.e., with Dirichlet boundary conditions transversal to the D-brane and Neumann boundary conditions along the D-brane

$$
\begin{aligned}
\mu=0, \ldots, p & \left.\partial_{\sigma} X^{\mu}\right|_{\sigma=0, \pi}=0, \\
\mu=p+1, \ldots, 9 & \left.\partial_{\tau} X^{\mu}\right|_{\sigma=0, \pi}=0 .
\end{aligned}
$$

where $(\sigma, \tau)$ denote the world-sheet space and time coordinates and $X^{\mu}$ the space-time coordinates. Their world-sheet superpartners are denoted as $\psi^{\mu}$ in the following. Upon quantization of an open string, the massless excitations $\psi_{-\frac{1}{2}}^{\mu}|0\rangle$ give rise to a $U(1)$ gauge field, which can only have momentum along the D-brane and is therefore confined to it. It is precisely the occurrence of these 


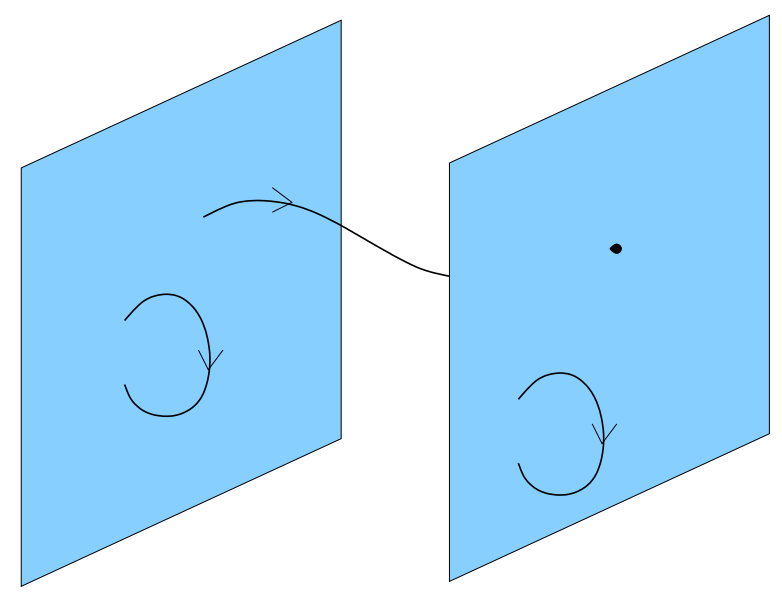

Figure 1: D-branes

gauge fields which makes D-branes interesting objects for string model building. It is quite remarkable that closed and open strings give rise to gravity and gauge theory, precisely the kind of forces we see in our universe.

Placing $N$ D-branes on top of each other the gauge fields on the branes transform in the adjoint representation of the gauge group $U(N)$. Can one also get $S O(N), S P(2 N), E_{6}, E_{7}, E_{8}, G_{2}$ or $F_{4}$ gauge symmetries? At least $S O(N)$ and $S P(2 N)$ gauge groups can be obtained from so called orientifolds (like Type I string theory). Please see [12] for a review.

\subsection{Orientifolds}

An orientifold is the quotient of Type II string theory by a discrete symmetry group $G$ including the world-sheet parity transformation $\Omega:(\sigma, \tau) \rightarrow(-\sigma, \tau)$. As a consequence the resulting string models contain non-oriented strings and their perturbative expansion also involves non-oriented surfaces like the Klein-bottle. Dividing out by such a symmetry, new objects called orientifold planes arise, whose presence can be detected for instance by computing the Klein-bottle amplitude

$$
K=\int_{0}^{\infty} \frac{d t}{t} \operatorname{Tr}\left(\frac{\Omega}{2} e^{-2 \pi t\left(L_{0}+\bar{L}_{0}\right)}\right) .
$$

Depending on which coordinate one defines as time, this amplitude can be viewed either as a closed string one loop amplitude or as a closed string propagating between two crosscaps $\left(\mathrm{RP}_{2}\right)$ (see figure 2 ). In the limit $l \rightarrow \infty$ one obtains infrared divergences, which result from non-vanishing one-point functions of massless fields on the crosscap. In other words, the crosscaps/orientifold planes do couple to some of closed string massless modes, i.e. they carry in particular charge under a higher rank R-R p-form $A_{p}$. In addition the coupling to gravity signals that the orientifold planes also carry tension. Such tadpoles for (higher rank) gauge fields $A_{p}$ are dangerous and indicate that one has not satisfied Gauß law. Recall that the equations of motion for a rank $p$ gauge fields read

$$
d \star F_{p+1}=\star j_{p}
$$




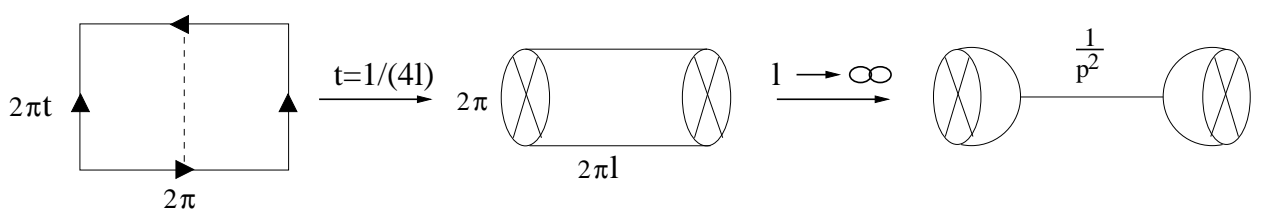

Figure 2: Klein bottle diagram

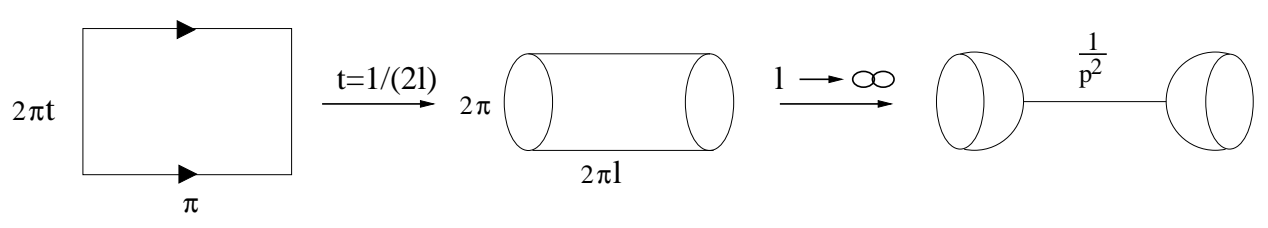

Figure 3: Annulus diagram

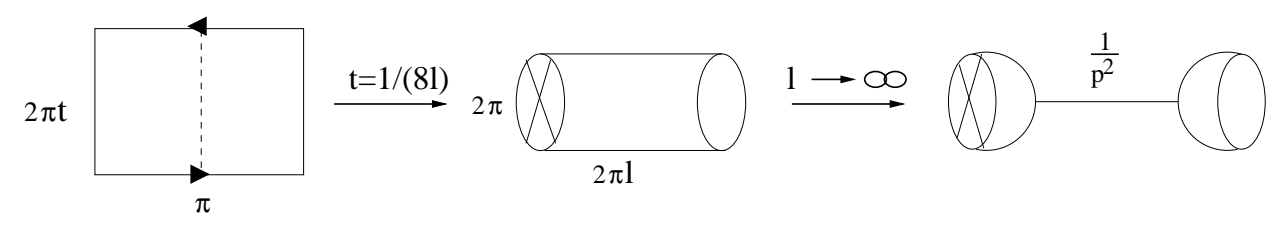

Figure 4: Möbius strip diagram

Integrating this equation over a compact space $\Sigma_{10-p}$ transversal to the $O p$ brane yields Gauß law

$$
0=\int_{\Sigma_{10-p}} d \star F_{p+1}=\int_{\Sigma_{10-p}} \star j_{p}=Q_{p}
$$

Therefore, the overall charge sitting on a compact space has to vanish.

In order to cancel the charge of the orientifold planes, other objects also carrying charge under the same p-forms have to be introduced. As we have seen already, these are precisely the $D_{p-1}$ branes, whose tadpole can be detected by computing the annulus amplitude as shown in Figure 3. In tree channel the annulus can be considered as a closed string propagating between two D-branes. The resulting infrared divergences arise again from non-vanishing massless tadpoles on the disc. To complete the story one also has to compute the non-oriented open string one-loop diagram, which is nothing else than the Möbius strip. In tree channel this can be seen as the a closed string propagating between a D-brane and an orientifold plane. To conclude for these orientifold models the introduction of D-branes is necessary in order to cancel all tadpoles and therefore they are the natural arena for consistent string model building with D-branes. In a consistent string background the global R-R tadpoles have to vanish.

Now, let us assume that $\mathcal{M}$ admits a complex structure so that we locally can introduce complex coordinates $z^{i}$. One can define the following orientifold models

- Type IIB: $\Omega \sigma$, where $\sigma: \mathcal{M} \rightarrow \mathcal{M}$ denotes a holomorphic involution, which is a symmetry of $\mathcal{M}$. 

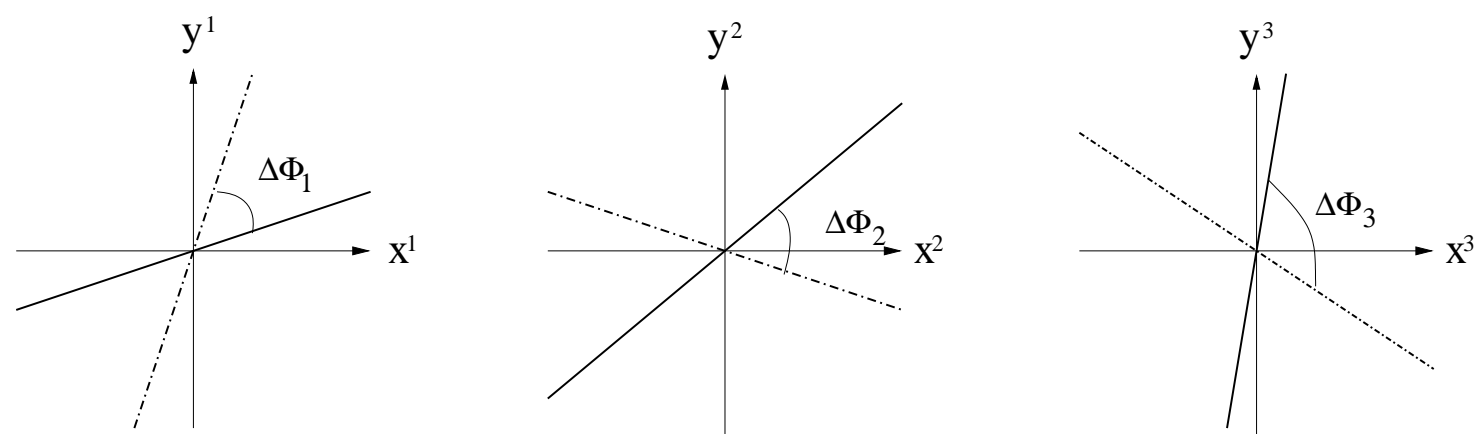

Figure 5: Intersecting D6-branes

- Type IIA: $\Omega \bar{\sigma}$, where $\bar{\sigma}: \mathcal{M} \rightarrow \mathcal{M}$ denotes an anti-holomorphic involution, which is a symmetry of $\mathcal{M}$.

\subsection{Chirality}

One of the main features of the Standard Model is that the light fermionic matter fields appear in chiral representations of the $S U(3)_{C} \times S U(2)_{W} \times U(1)_{Y}$ gauge symmetry such that all gauge anomalies are canceled. Considering just parallel D-branes in flat space one does not get chiral matter on the branes so that one has to invoke an additional mechanism to realize this phenomenologically very important feature. One way to do so is by allowing the branes to non-trivially intersect [13].

To be more precise consider two D6-branes sharing the four dimensional Minkowskian spacetime. This means that in the six dimensional transversal space the branes are three-dimensional and wrap a three dimensional cycle. In general position two such branes do intersect in a point in the internal space. Consider the simple case of a flat six-dimensional internal space. Choosing light cone gauge, let us introduce complex coordinates $z^{i}=x^{i}+i y^{i}$ with $i=0, \ldots, 3$. Then two D6-branes cover the $z^{0}$ plane and intersect in the other directions as shown in Figure 5 .

Placing for convenience one D-brane along the $x^{i}$ axes, an open string stretched between two intersecting D-branes has the following boundary conditions

$$
\begin{array}{cc}
\sigma=0: & \partial_{\sigma} X^{i}=\partial_{\tau} Y^{i}=0 \\
\sigma=\pi: & \partial_{\sigma} X^{i}+\tan \left(\Delta \Phi_{i}\right) \partial_{\sigma} Y^{i}=0 \\
& -\tan \left(\Delta \Phi_{i}\right) \partial_{\tau} X^{i}+\partial_{\tau} Y^{i}=0,
\end{array}
$$

which in complex coordinates read

$$
\begin{array}{ll}
\sigma=0: & \partial_{\sigma}\left(Z^{i}+\bar{Z}^{i}\right)=\partial_{\tau}\left(Z^{i}-\bar{Z}^{i}\right)=0 \\
\sigma=\pi: & \partial_{\sigma} Z^{i}+e^{2 i \Delta \Phi_{i}} \partial_{\sigma} \bar{Z}^{i}=0 \\
& \partial_{\tau} Z^{i}-e^{2 i \Delta \Phi_{i}} \partial_{\tau} \bar{Z}^{i}=0 .
\end{array}
$$

Now, implementing these boundary conditions in the mode expansion of the fields $Z^{i}$ and $\bar{Z}^{i}$, one finds [13]

$$
Z^{i}(\sigma, \tau)=\sum_{n \in \mathbb{Z}} \frac{1}{\left(n+\varepsilon_{i}\right)} \alpha_{n+\varepsilon_{i}}^{i} e^{-i\left(n+\varepsilon_{i}\right)(\tau+\sigma)}+\sum_{n \in \mathbb{Z}} \frac{1}{\left(n-\varepsilon_{i}\right)} \tilde{\alpha}_{n-\varepsilon_{i}}^{i} e^{-i\left(n-\varepsilon_{i}\right)(\tau-\sigma)}
$$


with $\varepsilon_{i}=\Delta \Phi_{i} / \pi$ for $i \in\{1,2,3\}$. Therefore the bosonic oscillator modes of the fields $Z^{1}, \ldots, Z^{3}$ are given by

$$
\alpha_{n+\varepsilon_{i}}^{i}, \quad \tilde{\alpha}_{n-\varepsilon_{i}}^{i} .
$$

Similarly, for the world-sheet fermions the modes are $\psi_{n+\varepsilon_{i}}^{i}$ and $\tilde{\psi}_{n-\varepsilon_{i}}^{i}$ in the R-R sector and with an additional 1/2-shift in the Neveu-Schwarz Neveu-Schwarz (NS-NS) sector. Therefore, in analogy to the closed string sector, an open string between two intersecting D-branes can be considered as a twisted open string. Since compared to the closed string sector the degrees of freedom in the open string sector are halfed, for generic intersections one gets one chiral Weyl fermion from the four-dimensional space-time point of view.

Summarizing, we have found that two generically intersecting D6-branes give rise to one chiral fermion at the intersection point. If we now consider the intersection between a stack of $M$ D6-branes with another stack of $N$ D6-branes it is clear that the, say,left-handed chiral fermion transforms in the bi-fundamental representation of the $U(M) \times U(N)$ gauge symmetry.

On a compact space two D-branes have in general a multiple intersection number so that on gets

\section{$I_{a b}$ chiral fermions at $(a, b)$ intersection}

where $I_{a b}$ denotes the topological intersection number between the two branes $a$ and $b$.

\subsection{T-duality}

Performing a T-duality in the $y^{I}$ directions one exchanges Neumann and Dirichlet boundary condition

$$
\begin{array}{ll}
\sigma=0: & \partial_{\sigma} X^{i}=\partial_{\sigma} Y^{i}=0 \\
\sigma=\pi: & \partial_{\sigma} X^{i}+\tan \left(\Delta \Phi_{i}\right) \partial_{\tau} Y^{i}=0 \\
& -\tan \left(\Delta \Phi_{i}\right) \partial_{\tau} X^{i}+\partial_{\sigma} Y^{i}=0
\end{array}
$$

with $\tan \left(\Delta \Phi_{i}\right)=\frac{m^{I}}{n^{I}} \frac{1}{R_{1}^{I} R_{2}^{I}}$. Therefore one gets a space-time filling D-brane with mixed boundary conditions, i.e. there exist a constant magnetic gauge flux $F^{I}=\tan \left(\Delta \Phi_{i}\right)=\frac{m^{I}}{n^{I}} \frac{1}{R_{1}^{I} R_{2}^{I}}$ on the brane world-volume. Here $n^{I}$ denotes the wrapping number of the brane around $T^{2}$ and $m^{I}$ the first Chern class of the gauge bundle

$$
c_{1}(F)=n^{I} \int_{T^{2}} F^{I}=m^{I}
$$

Therefore, for string model building the following two set-ups are equivalent:

\section{Orientifolds of Type IIA with intersecting D6-branes}

Orientifolds of Type IIB with magnetised D9-branes.

\subsection{Intersecting D-brane models}

The following presentation is in the spirit of mainly [14]. Consider Type IIA string theory on $\mathbb{R}_{1,3} \times C Y_{3}$ and perform an orientifold $\Omega \bar{\sigma}(-1)^{F_{L}}$ such that

$$
\bar{\sigma}(J)=-J
$$




$$
\bar{\sigma}\left(\Omega_{3}\right)=e^{2 i \varphi} \bar{\Omega}_{3} .
$$

The resulting $O 6$ plane preserves $\mathcal{N}=1$ supersymmetry, as the fixed point locus of an antiholomorphic involution defines a so-called special Lagrangian 3-cycle (sLag).

A three-cycle $\pi_{a}$ is called Lagrangian if the restriction of the Kähler form on the cycle vanishes

$$
\left.J\right|_{\pi_{a}}=0
$$

If the three-cycle in addition is volume minimizing, which can be expressed as the property that the imaginary part of the three-form $\Omega_{3}$ vanishes when restricted to the cycle,

$$
\left.\mathfrak{I}\left(e^{i \varphi_{a}} \Omega_{3}\right)\right|_{\pi_{a}}=0
$$

then the three-cycle is called special Lagrangian. The parameter $\varphi_{a}$ determines which $\mathcal{N}=1$ supersymmetry is preserved by the brane. Thus, different branes with different values for $\varphi_{a}$ preserve different $\mathcal{N}=1$ supersymmetries. One can show that 1.14 implies that the volume of the three-cycle is given by

$$
\operatorname{Vol}\left(\pi_{a}\right)=\left|\int_{\pi_{a}} \Re\left(e^{i \varphi_{a}} \Omega_{3}\right)\right| .
$$

The $O 6$ plane carries NS-NS and R-R tadpoles, which have to be canceled by introducing stacks of D6-banes wrapping 3-cycles $\pi_{a} \in H_{3}(\mathcal{M})$ and their orientifold images $\pi_{a}^{\prime}$. The resulting equation of motion for the R-R field strength $G_{8}=d C_{7}$ is

$$
\frac{1}{\kappa^{2}} d \star G_{8}=\mu_{6} \sum_{a} N_{a} \delta\left(\pi_{a}\right)+\mu_{6} \sum_{a} N_{a} \delta\left(\pi_{a}^{\prime}\right)-4 \mu_{6} \delta\left(\pi_{\mathrm{O} 6}\right),
$$

where $\delta\left(\pi_{a}\right)$ denotes the Poincare dual three-form of $\pi_{a}$. Since the left hand side in eq. (1.16) is exact, the R-R tadpole cancellation condition boils down to just a simple condition on the homology classes

$$
\sum_{a} N_{a}\left(\pi_{a}+\pi_{a}^{\prime}\right)-4 \pi_{O 6}=0
$$

The above condition implies that the overall three-cycle all the D-branes and orientifold planes wrap is trivial in homology. These conditions are augmented by additional K-theory constraints, whose field theory manifestation is the vanishing of the global $S U(2)$ Witten anomaly [15].

In order to preserve a global $\mathcal{N}=1$ supersymmetry all D6-branes have wrap special Lagrangian 3-cycles with $\varphi_{a}=\varphi_{06}$. In this case also all NS-NS tadpole vanish.

If the image cycle $\pi_{a}^{\prime}$ is point-wise identical to $\pi_{a}$, then one gets $S O / S P$ gauge symmetries, whereas in the general case $\pi_{a}^{\prime} \neq \pi_{a}$ one finds a $U\left(N_{a}\right)$ gauge symmetry. For a gauge group $G=$ $\prod_{a=1}^{K} U\left(N_{a}\right)$ the chiral massless spectrum is given in Table 1.

Open strings stretched between a D-brane and its $\bar{\sigma}$ image are the only ones left invariant under the combined operation $\Omega \bar{\sigma}(-1)^{F_{L}}$. Therefore, they transform in the antisymmetric or symmetric representation of the gauge group, indicating that the price we have to pay by considering intersecting D-branes in an orientifold background is that more general representations are possible for the chiral fermions. 
Table 1: Chiral spectrum for intersecting D6-branes

\begin{tabular}{|c|c|}
\hline Representation & Multiplicity \\
\hline$\square a$ & $\frac{1}{2}\left(\pi_{a}^{\prime} \circ \pi_{a}+\pi_{\mathrm{O} 6} \circ \pi_{a}\right)$ \\
$\square \square a$ & $\frac{1}{2}\left(\pi_{a}^{\prime} \circ \pi_{a}-\pi_{\mathrm{O} 6} \circ \pi_{a}\right)$ \\
$\left(\square_{a}, \square_{b}\right)$ & $\pi_{a} \circ \pi_{b}$ \\
$\left(\square_{a}, \square_{b}\right)$ & $\pi_{a}^{\prime} \circ \pi_{b}$ \\
\hline
\end{tabular}

Let us check that the R-R tadpole cancellation condition (1.17) together with Table 1 guarantees the absence of non-Abelian gauge anomalies.

$$
\begin{aligned}
\mathcal{A}= & -\sum_{b \neq a} N_{b}\left(\pi_{a} \circ \pi_{b}\right)+\sum_{b \neq a} N_{b}\left(\pi_{a}^{\prime} \circ \pi_{b}\right)+\frac{N_{a}-4}{2}\left(\pi_{a}^{\prime} \circ \pi_{a}+\pi_{O 6} \circ \pi_{a}\right)+ \\
& \frac{N_{a}+4}{2}\left(\pi_{a}^{\prime} \circ \pi_{a}-\pi_{O 6} \circ \pi_{a}\right) \\
= & -\pi_{a} \circ\left(\sum_{b} N_{b}\left(\pi_{b}+\pi_{b}^{\prime}\right)-4 \pi_{O 6}\right)=0 .
\end{aligned}
$$

Naively, there exist Abelian and mixed Abelian, non-Abelian anomalies, as well as gravitational anomalies. However, these are canceled by a generalized Green-Schwarz mechanism.

To the end of this lecture let me list some general comments on model building using intersecting/magnetized D-branes (for references please see [8]:

- Many concrete models have been discussed in the literature, where in most cases $\mathcal{M}$ was chosen to be a toroidal orbifold space $T^{6} / \mathbb{Z}_{N}$.

- On more general Calabi-Yau's not very much is known about special Lagrangian 3-cycles, but instead sometimes one can use pure conformal field theory techniques to construct such models $\rightarrow$ orientifolds of Gepner models.

- So far there are some semi-realistic MSSM like models, but no completely satisfactory model has emerged yet which also fulfils the second order requirements. Moreover, in most cases one gets exotic gauge factors and extra matter.

- Methods have been developed to compute the low-energy effective action for supersymmetric models, which is determined by the Kähler potential, the holomorphic gauge kinetic function and the superpotential.

\section{Flux compactifi cations}

Besides these model building techniques involving intersecting/magnetized D-branes a second very interesting development has occurred. Namely, it was realized that so-called flux compactifications have a very rich structure and in particular allow one to freeze some of the extra moduli (massless fields), which notoriously appear in string compactifications. This has developed into a very broad subject, so that here we will explain the main aspects using the example of three-form fluxes in Type IIB string theory as discussed in [16, 17]. 
But first let us recall that superstring theory contains many $\mathrm{p}$-form gauge fields $C_{p}$ in its massless spectrum. In particular, Type IIB contains NS-NS 0,2-forms and R-R 2,4-forms and their magnetic dual $8-p$-forms. Now, one considers solutions to the ten-dimensional string equations of motion with non-trivial fluxes $F_{p+1}=d C_{p} \neq 0$. The qualitative consequences of these fluxes are:

- The flux can generate new tadpoles for some the other p-form fields via Chern-Simons terms in the 10D action.

- The kinetic term

$$
S_{k i n}=\int_{\mathscr{M}} F \wedge \star F
$$

induces a scalar potential in the four dimensional effective action, which in general depends on the moduli controlling the size of the cycles $\Gamma_{p+1}$ the flux is running through, i.e.

$$
\int_{\Gamma_{p+1}} F_{p+1} \neq 0
$$

This can lead to moduli stabilisation.

- $F_{p+1}$ provides an extra source term in the Einstein equation

$$
R_{\mu v}-\frac{1}{2} g_{\mu v} R=8 \pi G T_{\mu v}\left(F_{p+1}\right)
$$

implying that the metric is not any longer Ricci-flat.

\subsection{New tadpoles}

The ten-dimensional Type IIB low-energy effective action reads

$$
\begin{aligned}
S_{I I B}=\frac{1}{\kappa_{10}^{2}} \int d^{10} x \sqrt{-g} & \left(R-\frac{\partial_{\mu} \tau \partial^{\mu} \bar{\tau}}{2(\operatorname{Im} \tau)^{2}}-\frac{G_{3} \cdot \bar{G}_{3}}{2 \cdot 3 ! \operatorname{Im} \tau}-\frac{\widetilde{F}_{5}^{2}}{4 \cdot 5 ! \operatorname{Im} \tau}\right) \\
& +\frac{1}{\kappa_{10}^{2}} \int \frac{C_{4} \wedge G_{3} \wedge \bar{G}_{3}}{4 i \operatorname{Im} \tau}+\text { local sources }
\end{aligned}
$$

with the dilaton-axion field $\tau=C_{0}+i e^{-\varphi}$, the complex three-form flux $G_{3}=F_{3}-\tau H_{3}$ and the self-dual 5-form field $\widetilde{F}_{5}=F_{5}-\frac{1}{2} C_{2} \wedge H_{3}+\frac{1}{2} F_{3} \wedge B_{2}$. The Bianchi identity for this fields reads

$$
d \widetilde{F}_{5}=d \star \widetilde{F}_{5}=H_{3} \wedge F_{3}+2 \kappa^{2} \mu_{3} \rho_{\text {local }},
$$

which means that the $G_{3}$-flux contributes to the D3-brane tadpole cancellation condition by an amount

$$
N_{\text {flux }}=\frac{1}{\left(4 \pi^{2} \alpha^{\prime}\right)^{2}} \int_{\mathcal{M}} H_{3} \wedge F_{3}
$$




\subsection{The scalar potential}

The tree level scalar potential in four dimensions is given by (suppressing the warp factor)

$$
V=\frac{1}{4 \kappa_{10}^{2} \operatorname{Im} \tau} \int_{\mathcal{M}} d^{6} y G_{3} \wedge \star_{6} \bar{G}_{3} .
$$

One can split the 3-form flux into an imaginary self-dual piece $G^{+}$and an imaginary anti-self-dual piece $G^{-}$, i.e.

$$
\star_{6} G_{3}^{ \pm}= \pm i G_{3}^{ \pm} .
$$

Using $\star_{6}^{2}=-1$ after some little algebra one writes the scalar potential as

$$
V=\frac{1}{2 \kappa_{10}^{2} \operatorname{Im} \tau} \int_{\mathcal{M}} d^{6} y G_{3}^{\mp} \wedge \star_{6} \bar{G}_{3}^{\mp} \mp \frac{i}{4 \kappa_{10}^{2} \operatorname{Im} \tau} \int_{\mathscr{M}} d^{6} y G_{3} \wedge \bar{G}_{3}
$$

where the second term $T_{\text {flux }}$ (including the sign) is proportional to $N_{\text {flux }}$. Now, one can distinguish the two cases

- $G_{3}^{-}=0, G_{3}$ ISD $\rightarrow N_{f l u x} \geq 0$ and $T_{f l u x} \geq 0$, i.e. the flux contributes like a $D 3$-brane to the NS-NS and R-R tadpole

- $G_{3}^{+}=0, G_{3}$ IASD $\rightarrow N_{f l u x} \leq 0$ and $T_{f l u x} \geq 0$, i.e. the flux contributes like a $\overline{D 3}$-brane to the NS-NS and R-R tadpole $\rightarrow$ supersymmetry breaking.

Consider now ISD fluxes. The first term in (2.9) is positive definite and can be written as an F-term

$$
V_{F}=e^{K}\left(\sum_{i \bar{j}} G^{i \bar{j}} D_{i} W D_{\bar{j}} \bar{W}-3|W|^{2}\right)
$$

of a superpotential $W[18]$. Here the sum runs over all moduli, i.e. the complex structure, the dilaton and the axion-volume modulus $\rho=b+i \operatorname{Vol}_{\mathcal{M}}$ whose Kähler potentials are given at tree level by

$$
K=-\log \left[-i \int_{\mathscr{M}} \Omega \wedge \bar{\Omega}\right]-\log [-i(\tau-\bar{\tau})]-3 \log [-i(\rho-\bar{\rho})]
$$

Moreover, $G^{i \bar{j}}$ is the inverse of the metric $G_{i \bar{j}}=\partial_{i} \partial_{\bar{j}} K$ and the covariant derivative is defined as $D_{i}=\partial_{i}+\partial_{i} K$. In our case the superpotential is of Gukov-Vafa-Witten type [19]

$$
W=\int_{\mathscr{M}} \Omega_{3} \wedge G_{3},
$$

which apparently depends only on the complex structure moduli and the dilaton. Since $W$ does not depend on the Kähler modulus $\rho$, the $\rho$ term in the sum in (2.10) cancels against the $-3|W|^{2}$ term, so that the scalar potential is of the so-called no-scale type

$$
V_{F}=e^{K}\left(\sum_{I \bar{J}} G^{I \bar{J}} D_{I} W D_{\bar{J}} \bar{W}\right) \geq 0
$$

where the sum only runs over the dilaton and the complex structure moduli. To summarize, the minima of the scalar potential can be characterized as follows: 
- $V_{F}=0 \rightarrow G$ ISD, $G=G^{(2,1)}+G^{(0,3)} \rightarrow D_{I} W=0$ for all $I$, freezes in general all complex structure and moduli and the dilaton.

- Supersymmetry $\rightarrow D_{\rho} W \simeq W=0 \rightarrow G=G^{(2,1)}$.

Let us end this lecture with some comments:

- In our considerations we neglected the backreaction of the flux on the geometry, i.e. the new source term in the Einstein equation. In a model with only D3 branes and G-fluxes this is just a warped Calabi-Yau geometry

$$
d s_{10}^{2}=e^{2 A(y)} g_{\mu \nu}^{(4)} d x^{\mu} d x^{v}+e^{-2 A(y)} \tilde{g}_{m n}^{(6)} d y^{m} d y^{n}
$$

with

$$
\widetilde{F}_{5}=(1+\star)\left[d \alpha(y) \wedge d \mathrm{Vol}^{4}\right]
$$

and $\alpha=\exp (4 A)$. For models with $D 7$ branes the geometry is not any longer of this simple warped form, but involves a strong backreaction, which is best described via F-theory on Calabi-Yau fourfolds.

- For supersymmetry also O3-planes are needed $\rightarrow$ orientifolds of Type IIB on Calabi-Yau manifolds

- For $H^{1}(\mathcal{M}) \neq 0$ one gets the additional supersymmetry condition that the flux has to be primitive, i.e. $J \wedge G=0$.

\section{The KKLT scenario}

We have seen that G-flux allows one to freeze all complex structure moduli and the dilaton. However, at this level the Kähler moduli remain as massless fields. Two mechanisms have been proposed to fix also these fields:

- There are $\alpha^{\prime}$ corrections to the Kähler potential, which imply $\alpha^{\prime}$ corrections to the scalar potential $V_{F}$.

- There can be non-perturbative string corrections to the superpotential generated by for instance stringy instantons.

The second scenario has been discussed by KKLT and their approach is going to be presented in this lecture [20].

The starting point is again the Type IIB string with G-flux and superpotential

$$
W=\int \Omega_{3} \wedge G_{3} .
$$

Recall that the Kähler potentials are given at tree level by

$$
K=-\log \left[-i \int_{\mathscr{M}} \Omega \wedge \bar{\Omega}\right]-\log [-i(\tau-\bar{\tau})]-3 \log [-i(\rho-\bar{\rho})]
$$

In order to fix also the Kähler moduli, KKLT proposed a two step process. 
- One turns on an ISD but non-supersymmetric G-flux $G=G^{(2,1)}+G^{(0,3)}$, so that $V_{F}=0$ but $W=W_{0}$. We assume that all complex structure moduli are frozen and that the dilaton is frozen in the perturbative regime $g_{s}<<1$.

- After integrating out the heavy complex structure fields one gets an effective theory just for Kähler moduli, where now non-perturbative corrections to the superpotential, as induced by for instance Euclidean D3-branes, are taken into account. (According to Witten, such corrections arise in the F-theory picture whenever the fourfold admits divisors of arithmetic genus one, i.e. $\sum_{i=0}^{3}(-1)^{i} H^{(0, i)}(D)=1$, which project to 4-cycles in the base of the elliptic fibration.) Similar corrections can be induced by gaugino condensation on D7-branes. Phenomenologically, one includes such corrections by the leading order instanton correction of the generic form

$$
W_{\text {inst }} \simeq e^{i a \rho}
$$

\subsection{AdS minima}

For the second step one simply makes the following phenomenological ansatz

$$
W=W_{0}+A e^{i a \rho}
$$

where for simplicity we assume $\rho=i \sigma$ and $A, a, W_{0} \in \mathbb{R}$. In the following we also assume that one finds non-supersymmetric minima of $V_{F}$ with $W_{0}<0$.

Using (3.4) one looks for supersymmetric minima in $\sigma$, i.e. solutions to the equation $D_{\sigma} W=0$. This yields the condition

$$
W_{0}=-A e^{-a \sigma_{c r}}\left(1+\frac{2}{3} a \sigma_{c r}\right) .
$$

The value of the scalar potential at this new supersymmetric minimum $\sigma_{c r}$ is given by

$$
V_{0}=\frac{-a^{2} A^{2} e^{-2 a \sigma_{c r}}}{6 \sigma_{c r}}<0
$$

Self-consistency of the solution requires $\sigma_{c r}>>1$ (no $\alpha^{\prime}$ corrections) and $a \sigma_{c r}>>1$ (leading order instanton approximation justified). In Figure 6 the resulting scalar potential is shown, which apparently has a stable self-consistent AdS minimum.

\section{2 dS minima}

Concerning moduli stabilisation we have already reached the goal, however KKLT realized that with additional stringy input this scenario can also lead to (non-supersymmetric) de-Sitter solutions. The new ingredient is given by the introduction of $\overline{D 3}$ branes respectively D7-branes with magnetic flux.

- $\overline{D 3}$ branes in an ISD $G$-flux background are stuck at the point of maximal warping. Put differently, ISD G-flux induces susy soft masses for the brane moduli of $\overline{D 3}$ branes. 


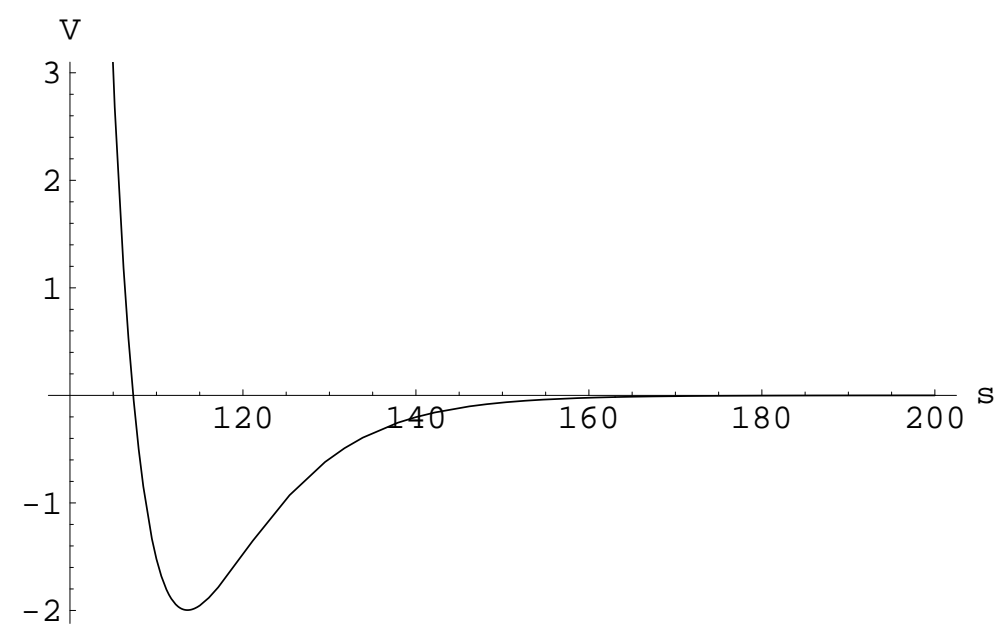

Figure 6: The scalar potential $\left(10^{15} \cdot V(\sigma)\right)$ for the choice $A=1, a=0.1, W_{0}=-10^{-4}$

- We have to satisfy the R-R tadpole cancellation condition $N_{f l u x}+Q_{\overline{D 3}}=Q_{O 3}$, which gives the NS-NS tadpole (tension)

$$
V_{D}=N_{\text {flux }}+T_{\overline{D 3}}-T_{O 3}=2 T_{\overline{D 3}} .
$$

Taking also the warping of the geometry into account this tension can be determined to be

$$
V_{D}=\frac{8 D}{\sigma^{2}}
$$

so that the total scalar potential now reads

$$
V_{t o t}=V_{F}+V_{D}=\frac{a A e^{-a \sigma}}{2 \sigma^{2}}\left(\frac{1}{3} \sigma a A e^{-a \sigma}+W_{0}+A e^{-a \sigma}\right)+\frac{D}{\sigma^{2}}
$$

By tuning of $D$ the local minimum of $V_{t o t}$ is shifted to positive values and one finally gets metastable de-Sitter vacua. In Figure 7 the resulting scalar potential is shown, which apparently leads to a meta-stable dS vacuum with small cosmological constant. Again to the end of this lecture a few comments:

- As it is discussed so far, the KKLT set-up is just a possible scenario and not yet a concrete model. It remains to be seen whether one can construct concrete string models which are sufficiently under control to confirm this scenario.

- The realization that flux compactifications generically lead to local minima fixing all moduli motivated to the so-called landscape picture of string theory, which seems to indicate that there exists an immense number of (meta-) stable solutions to the string equations of motion (we will come back this in final lecture). 


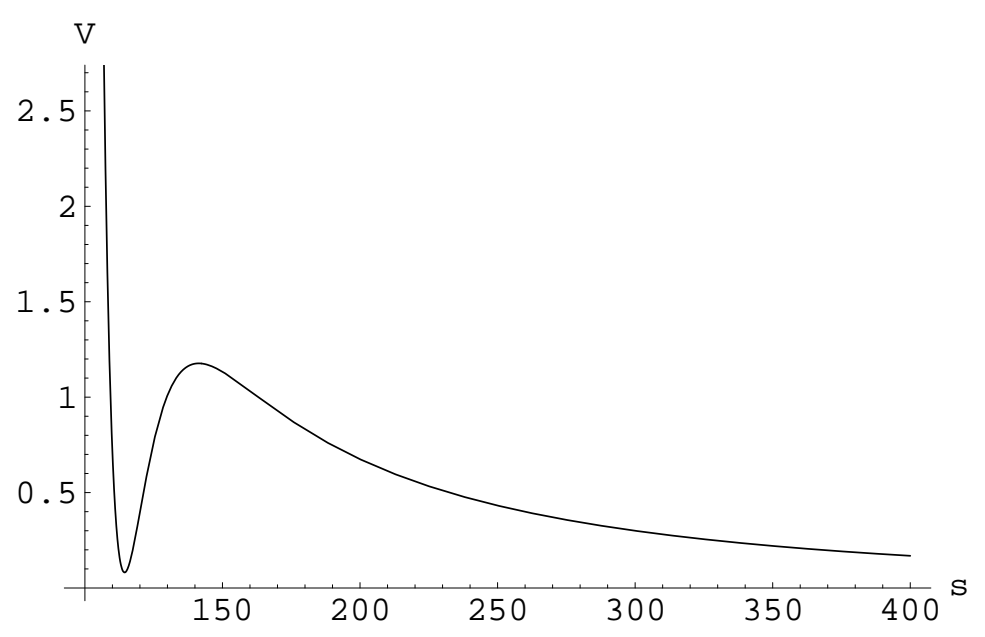

Figure 7: The scalar potential $\left(10^{15} \cdot V(\sigma)\right)$ for the choice $A=1, a=0.1, W_{0}=-10^{-4}, D=2.7 \cdot 10^{-11}$

\section{Toward realistic brane and flux compactifi cations}

A natural step is to combine the ideas of flux compactifications with the methods for constructing quasi-realistic string models on intersecting/magnetised D-branes. The aim is to construct chiral semi-realistic Standard-like models with partially frozen moduli.

As a concrete example consider the Type IIB $\Omega R(-1)^{F_{L}}$ orientifold on

$$
\mathcal{M}=\frac{T^{6}}{\mathbb{Z}_{2} \times \mathbb{Z}_{2}}
$$

where $R$ reflects all six internal directions and the two $\mathbb{Z}_{2}$ actions are given by

$$
\Theta:\left\{\begin{array}{l}
z_{1} \rightarrow-z_{1} \\
z_{2} \rightarrow-z_{2}, \\
z_{3} \rightarrow z_{3}
\end{array} \quad \Theta^{\prime}:\left\{\begin{array}{l}
z_{1} \rightarrow z_{1} \\
z_{2} \rightarrow-z_{2} \\
z_{3} \rightarrow-z_{3} .
\end{array}\right.\right.
$$

Depending on the discrete torsion between these two $\mathbb{Z}_{2}$ operations the resulting orbifold has either $\left(h_{21}, h_{11}\right)=(51,3)$ or $\left(h_{21}, h_{11}\right)=(3,51)$. Here we consider the first of these two models, which has 83 -cycles in the untwisted sector and 323 -cycles in each of the three $\mathbb{Z}_{2}$ twisted sectors. Following [21, 22, 23, 24], in order to apply the general methods introduced in the first lectures, let us dwell upon this example in more detail.

\subsection{A supersymmetric $G_{3}$ flux}

The fluxes $F_{3}$ and $H_{3}$ obey the Bianchi identity and take values in $H^{3}(M, \mathbb{Z})$, i.e.

$$
\frac{1}{(2 \pi)^{2} \alpha^{\prime}} \int_{M} H_{3} \in N_{\min } \mathbb{Z}, \quad \frac{1}{(2 \pi)^{2} \alpha^{\prime}} \int_{M} F_{3} \in N_{\min } \mathbb{Z}
$$

where $N_{\min }$ is an integer which guarantees that in orbifold models we only turn on untwisted 3form fluxes, for which we can trust the supergravity approximation. Taking also the orientifold projection into account in the $T^{6} / \mathbb{Z}_{2} \times \mathbb{Z}_{2}$ orbifold one gets $N_{\min }=8$. 
Due to the $\mathbb{Z}_{2} \times \mathbb{Z}_{2}$ action the complex structure of $T^{6}$ is partially fixed, so that $T^{6}=T^{2} \times$ $T^{2} \times T^{2}$. Defining on each $T^{2}$ factor complex coordinates

$$
z^{I}=x^{I}+\tau^{I} y^{I}
$$

a symplectic basis of $H^{3}(\mathcal{M}, \mathbb{Z})$ is given by

$$
\begin{aligned}
\alpha_{0} & =d x^{1} \wedge d x^{2} \wedge d x^{3}, & & \alpha_{i}=(-1)^{i-1} d y^{i} \wedge d x_{j} \wedge d x_{k}, \\
\beta^{i} & =(-1)^{i} d x^{i} \wedge d y_{j} \wedge d y_{k}, & & \beta^{0}=-d y^{1} \wedge d y^{2} \wedge d y^{3}
\end{aligned}
$$

with $i \neq j \neq k \neq i$ and $j<k$. These 3 -forms satisfy

$$
\int_{\mathcal{M}} \alpha_{I} \wedge \beta^{J}=\delta_{I J}
$$

In addition the covariantly constant holomorphic 3-form can be expressed as

$$
\begin{aligned}
\Omega_{3}=d z_{1} \wedge d z_{2} \wedge d z_{3} & =\alpha_{0}+\tau_{i} \alpha_{i}-\beta^{i} \tau_{j} \tau_{k}+\tau_{1} \tau_{2} \tau_{3} \beta^{0} \\
& =X^{\Lambda} \alpha_{\Lambda}-F_{\Lambda} \beta^{\Lambda}
\end{aligned}
$$

Now, choosing the G-flux to be

$$
\begin{aligned}
F_{3} & =\alpha_{0}+\beta^{0} \\
H_{3} & =\alpha_{0}-\sum_{i} \alpha_{i}-\sum_{i} \beta^{i}-2 \beta^{0}
\end{aligned}
$$

the resulting superpotential can be expressed as

$$
\begin{aligned}
W & =\int G \wedge \Omega_{3} \\
& =(1-\tau) \tau_{1} \tau_{2} \tau_{3}-\tau\left(\tau_{1} \tau_{2}+\tau_{2} \tau_{3}+\tau_{1} \tau_{3}\right)-\tau\left(\tau_{1}+\tau_{2} \tau_{3}\right)-(1+2 \tau) .
\end{aligned}
$$

Since the G-flux is totally symmetric with respect to the three $T^{2}$ factors, we can set $\tau_{1}=\tau_{2}=\tau_{3}=t$ and try to solve the supersymmetry conditions $W=\partial W / \partial \tau=\partial W / \partial t=0$. One finds that the solution to these three equations in two variables is

$$
t=\tau=e^{\frac{2 \pi i}{3}}
$$

At this point the G-flux can be written as

$$
\frac{1}{(2 \pi)^{2} \alpha^{\prime}} G=\frac{8(1-\tau)}{\sqrt{3}}\left(d \bar{z}_{1} d z_{2} d z_{3}+d z_{1} d \bar{z}_{2} d z_{3}+d z_{1} d z_{2} d \bar{z}_{3}\right)
$$

which apparently is indeed of type $G^{(2,1)}$ and yields a contribution $N_{\text {flux }} / 4=48$ to the D3-brane tadpole cancellation condition. 


\subsection{MSSM like magnetized D-branes}

In order to cancel the resulting tadpoles, one introduces magnetised D9-branes, which are T-dual to the intersecting D6-branes. Such a magnetised brane is characterised by three pairs of integers $\left(n_{a}^{I}, m_{a}^{I}\right)$ which satisfy

$$
\frac{m_{a}^{I}}{2 \pi} \int_{T_{I}^{2}} F_{a}^{I}=n_{a}^{I}
$$

where the $m_{a}^{I}$ denote the wrapping number of the D9-brane around the torus $T_{I}^{2}$ and $n_{a}^{I}$ is the magnetic flux. The orientifold projection acts as follows on these quantum numbers $\Omega R(-1)^{F_{L}}$ : $\left(n_{a}^{I}, m_{a}^{I}\right) \rightarrow\left(n_{a}^{I},-m_{a}^{I}\right)$. Since $h_{11}=3$ one gets in the orientifold four tadpole cancellation conditions

$$
\begin{aligned}
\sum_{a} N_{a} n_{a}^{1} n_{a}^{2} n_{a}^{3} & =8-\frac{N_{\text {flux }}}{4} \\
\sum_{a} N_{a} n_{a}^{I} m_{a}^{J} m_{a}^{K} & =-8 \quad \text { for } I \neq J \neq K \neq I .
\end{aligned}
$$

In order for each brane to preserve the same supersymmetry as the orientifold planes, they have to satisfy

$$
\sum_{I} \arctan \left(\frac{m_{a}^{I} \mathcal{K}^{I}}{n_{a}^{I}}\right)=0
$$

where $\mathcal{K}^{I}$ denotes the volume of the I-th torus $T^{2}$ in units of $\alpha^{\prime}$. The number of chiral fermions between two different magnetised branes is given by the index

$$
I_{a b}=\prod_{I}\left(n_{a}^{I} m_{b}^{I}-m_{a}^{I} n_{b}^{I}\right)
$$

and can lead to matter in bifundamental, symmetric or anti-symmetric representations of the gauge group.

Taking the flux quantisation with $N_{\min }=8$ into account, the contribution of the flux to the D3-brane tadpole is given by $N_{\text {flux }} / 4 \in 16 \mathbb{Z}$. Therefore, for non-trivial flux the right hand side of the D3-brane tadpole cancellation condition (4.13) is always negative.

Consider for instance the magnetised brane $\left(n_{a}^{I}, m_{a}^{I}\right)=(-2,1)(-3,1)(-4,1)$, which is supersymmetric for

$$
\arctan \left(A_{1} / 2\right)+\arctan \left(A_{2} / 3\right)+\arctan \left(A_{3} / 4\right)=\pi
$$

and contributes as $(-24,-4,-2,-3)$ to the four tadpole conditions and can therefore compensate the negative contributions of the four $O$-planes and the flux.

Introducing the supersymmetric magnetised branes shown in Table 2 [23] cancels all the tadpole and gives rise to a one-generation MSSM-like model with gauge group

$$
G=S U(3) \times S U(2) \times S U(2) \times U(1)_{B-L} \times\left[U(1)^{\prime} \times U S p(8)\right] .
$$

Supersymmetry enforces $A_{2}=A_{3}$. For more technical and phenomenological details of such models please consult the original literature. Note that the branes $b, c$ can be placed directly on top the 
Table 2: Wrapping numbers for semi-realistic model.

\begin{tabular}{|c||c|c|c|}
\hline$N_{a}$ & $\left(n_{a}^{1}, m_{a}^{1}\right)$ & $\left(n_{a}^{2}, m_{a}^{2}\right)$ & $\left(n_{a}^{3}, m_{a}^{3}\right)$ \\
\hline \hline$N_{a}=3$ & $(1,0)$ & $(1,1)$ & $(1,-1)$ \\
\hline$N_{b}=1$ & $(0,1)$ & $(1,0)$ & $(0,-1)$ \\
\hline$N_{c}=1$ & $(0,1)$ & $(0,-1)$ & $(1,0)$ \\
\hline$N_{d}=1$ & $(1,0)$ & $(1,1)$ & $(1,-1)$ \\
\hline \hline$N_{h_{1}}=1$ & $(-2,1)$ & $(-3,1)$ & $(-4,1)$ \\
\hline$N_{h_{2}}=1$ & $(-2,1)$ & $(-4,1)$ & $(-3,1)$ \\
\hline$N_{f}=4$ & $(1,0)$ & $(1,0)$ & $(1,0)$ \\
\hline
\end{tabular}

corresponding $O 7$-planes yielding a gauge group $S U(2) \times S U(2)$. This example shows that it is indeed possible to construct supersymmetric semi-realistic string models with fluxes and partially frozen moduli. This is an encouraging observation, but of course much more work is needed to really establish an entire class of such models.

As customary, let me end this lecture with some comments:

- In our considerations we neglected the backreaction of the magnetized D9-branes.

- Three generation MSSM like models are possible with supersymmetry breaking fluxes (i.e. $\left.G^{(0,3)} \neq 0\right)$. The backreaction of this flux component induces soft supersymmtry breaking terms on the magnetised D9-branes.

\section{Statistics of string vacua}

We have seen that flux compactifications lead to moduli stabilisation, thereby giving rise to many in general discrete (non-)supersymmetric vacua, for which the low energy effective action is different. In fact, fluxes reduce the continuous moduli space of vacua to a countable discretuum. One may ask:

\section{How many flux vacua are there?}

Following [25], let us make an estimate. Consider Type IIB compactified on a Calabi-Yau manifold with $b_{3} 3$-cycles $\alpha^{i}$. Now we turn on general G-flux through these 3-cycles

$$
\frac{1}{(2 \pi)^{2} \alpha^{\prime}} \int_{M} H_{3}=\sum_{i} N_{N S}^{i} \alpha_{i}, \quad \frac{1}{(2 \pi)^{2} \alpha^{\prime}} \int_{M} F_{3}=\sum_{i} N_{R}^{i} \alpha_{i},
$$

so that

$$
N_{\text {flux }}=\frac{1}{(2 \pi)^{4} \alpha^{\prime 2}} \int F_{3} \wedge H_{3}=\eta_{i j} N_{N S}^{i} N_{R}^{j}>0
$$

with $\eta_{i j}=\int \alpha_{i} \wedge \alpha_{j}$. The tadpole cancellation condition reads

$$
\frac{N_{\text {flux }}}{2}+N_{D-\text { branes }}=L_{*}
$$


where $L_{*}$ denotes the contribution of the orientifold planes. Now, we want to count the number of solutions with $0 \leq L \leq L_{*}$ with $L=N_{\text {flux }} / 2$. Following [26, 25] this number is given by

$$
\begin{aligned}
\mathcal{N}_{\text {flux }}\left(L \leq L_{*}\right) & =\sum_{\text {susy vac }} \theta\left(L_{*}-L\right) \\
& =\sum_{\text {vac }} \frac{1}{2 \pi i} \int_{C} \frac{d \alpha}{\alpha} e^{\alpha\left(L_{*}-L\right)} \\
& =\frac{1}{2 \pi i} \int_{C} \frac{d \alpha}{\alpha} e^{\alpha L_{*}}\left(\sum_{\text {vac }} e^{-\frac{\alpha}{2} \mathbf{N} \eta \mathbf{N}}\right)
\end{aligned}
$$

where the path $C$ in the complex plane runs parallel to the $y$ axis with small positive $x$. After approximating the discrete sum over the flux quanta by an integral, the sum over all vacua can be written as

$$
\begin{aligned}
\mathcal{N}(\alpha) & =\sum_{v a c} e^{-\frac{\alpha}{2} \mathbf{N} \eta \mathbf{N}} \\
& =\int_{M} d^{2 m} z \int d^{4 m} N e^{-\frac{\alpha}{2} \mathbf{N} \eta \mathbf{N}} \delta^{2 m}(D W)\left|\operatorname{det} D^{2} W\right|
\end{aligned}
$$

with $m=b_{3} / 2$ and $M$ denoting a fundamental region in the complex structure/dilaton moduli space. However, the scaling of the number of vacua with $L_{*}$ can be estimated without evaluating this integral. Let us rescale $N \rightarrow N / \sqrt{\alpha}$, which implies $\mathcal{N}(\alpha) \rightarrow \alpha^{-2 m} \mathcal{N}(1)$ so that gets

$$
\begin{aligned}
N_{\text {flux }}\left(L \leq L_{*}\right) & =\frac{1}{2 \pi i} \int_{C} \frac{d \alpha}{\alpha^{2 m+1}} e^{\alpha L_{*}} \mathcal{N}(1) \\
& =\theta\left(L_{*}\right) \frac{L_{*}^{2 m}}{(2 m) !} \mathcal{N}(1) .
\end{aligned}
$$

This is good approximation as long as the radius of the sphere in $N$-space is large enough, i.e. $L_{*} \gg 2 m$. For typical numbers such as $L_{*} \simeq 1000$ (as they appear in F-theory) and $m \simeq 200$ one gets $\mathcal{N}_{\text {flux }} \simeq 10^{250}$. This is an amazingly large number, which as we will discuss in a moment, sheds some new light on the string vacuum problem.

In [26] the integral (5.5) was evaluated further (with $\left|\operatorname{det} D^{2} W\right| \rightarrow \operatorname{det} D^{2} W$ ) leading eventually to the formula

$$
N_{\text {flux }}\left(L \leq L_{*}\right)=\frac{2 \pi L_{*}^{2 m}}{\pi^{n}(2 m) !} \int_{\mathcal{F} \times \mathcal{H}} \operatorname{det}(-R-\omega)
$$

where $\mathcal{F}$ denotes the fundamental region of $S L(2, \mathbb{Z}), \mathcal{H}$ the fundamental region of the complex structure moduli space and $R$ and $\omega$ the curvature and Kähler two forms.

In view of this huge vacuum degeneracy, the so-called string landscape, M.R. Douglas has proposed a statistical approach to the string vacuum problem [27]. In its pragmatic version it says that complementary to a (necessary) model by model search one should study the statistical distribution of various physical quantities in the ensemble of string vacua. Such an approach might be helpful to

- estimate the frequency with which standard-like string models arise

- get an idea in which regions of the landscape to look for realistic models 
- find statistical evidence that standard-like properties are extremely rare, i.e. almost excluded $\rightarrow$ falsification of string theory

- argue for a uniform distribution of certain physical quantities like for instance the cosmological constant, shedding a new light on so-called fine tuning problems

More philosophically, one can also combine the landscape picture with the weak anthropic principle, saying that in some meta-world all string theory vacua are realized and from the many possibilities, we of course happen to live in a (meta-)stable one where the physical parameters have just the right values to bring about almost intelligent life forms. This might explain why some anthropically essential physical quantities like the cosmological constant have the "fine tuned" value we observe.

Of course no final word has been spoken in this matter and one should carry on investigating the set of string vacua with all possible means.

Acknowledgements: I would like to thank the organizers of the RTN Winter School on Strings, Supergravity and Gauge Theories to invite me to present these lectures. I would like to thank Timo Weigand for useful comments about the manusscript. 


\section{References}

[1] A. M. Uranga, "Chiral four-dimensional string compactifications with intersecting D-branes," Class. Quant. Grav. 20 (2003) S373-S394, hep-th/0301032.

[2] F. G. Marchesano, "Intersecting D-brane models," hep-th/0307252.

[3] T. Ott, "Aspects of stability and phenomenology in type IIA orientifolds with intersecting D6-branes," Fortsch. Phys. 52 (2004) 28-137, hep-th/0309107.

[4] E. Kiritsis, "D-branes in standard model building, gravity and cosmology," Fortsch. Phys. 52 (2004) 200-263, hep-th/0310001.

[5] L. Görlich, "N=1 and non-supersymmetric open string theories in six and four space-time dimensions," hep-th/0401040.

[6] D. Lüst, "Intersecting brane worlds: A path to the standard model?," Class. Quant. Grav. 21 (2004) S1399-1424, hep-th/0401156.

[7] R. Blumenhagen, "Recent progress in Intersecting D-brane models," hep-th/0412025.

[8] R. Blumenhagen, M. Cvetic, P. Langacker, and G. Shiu, "Toward realistic intersecting D-brane models," hep-th/0502005.

[9] J. Polchinski, "Dirichlet-Branes and Ramond-Ramond Charges," Phys. Rev. Lett. 75 (1995) 4724-4727, hep-th/9510017.

[10] J. Polchinski, S. Chaudhuri, and C. V. Johnson, "Notes on D-Branes," hep-th/9602052.

[11] J. Polchinski, "Lectures on D-branes," hep-th/9611050.

[12] C. Angelantonj and A. Sagnotti, “Open strings,” Phys. Rept. 371 (2002) 1-150, hep-th/0204089.

[13] M. Berkooz, M. R. Douglas, and R. G. Leigh, "Branes intersecting at angles," Nucl. Phys. B480 (1996) 265-278, hep-th/9606139.

[14] R. Blumenhagen, V. Braun, B. Körs, and D. Lüst, "Orientifolds of K3 and Calabi-Yau manifolds with intersecting D-branes," JHEP 07 (2002) 026, hep-th/0206038.

[15] E. Witten, “An SU(2) anomaly,” Phys. Lett. B117 (1982) 324-328.

[16] S. B. Giddings, S. Kachru, and J. Polchinski, "Hierarchies from fluxes in string compactifications," Phys. Rev. D66 (2002) 106006, hep-th/0105097.

[17] S. Kachru, M. B. Schulz, and S. Trivedi, "Moduli stabilization from fluxes in a simple IIB orientifold," JHEP 10 (2003) 007, hep-th/0201028.

[18] T. R. Taylor and C. Vafa, "RR flux on Calabi-Yau and partial supersymmetry breaking," Phys. Lett. B474 (2000) 130-137, hep-th/9912152.

[19] S. Gukov, C. Vafa, and E. Witten, “CFT's from Calabi-Yau four-folds,” Nucl. Phys. $\mathbf{B 5 8 4}$ (2000) 69-108, hep-th/9906070.

[20] S. Kachru, R. Kallosh, A. Linde, and S. P. Trivedi, "De Sitter vacua in string theory," Phys. Rev. D68 (2003) 046005, hep-th/0301240.

[21] R. Blumenhagen, D. Lüst, and T. R. Taylor, "Moduli stabilization in chiral type IIB orientifold models with fluxes," Nucl. Phys. B663 (2003) 319-342, hep-th/0303016.

[22] J. F. G. Cascales and A. M. Uranga, "Chiral 4d N = 1 string vacua with D-branes and NSNS and RR fluxes," JHEP 05 (2003) 011, hep-th/0303024. 
[23] F. Marchesano and G. Shiu, "MSSM vacua from flux compactifications," hep-th/0408059.

[24] F. Marchesano and G. Shiu, "Building MSSM flux vacua," JHEP 11 (2004) 041, hep-th/0409132.

[25] F. Denef and M. R. Douglas, "Distributions of flux vacua," JHEP 05 (2004) 072, hep-th/0404116.

[26] S. Ashok and M. R. Douglas, “Counting flux vacua,” JHEP 01 (2004) 060, hep-th/0307049.

[27] M. R. Douglas, “The statistics of string / M theory vacua," JHEP 05 (2003) 046, hep-th/ 0303194.

[28] J. F. G. Cascales and A. M. Uranga, "Chiral 4d string vacua with D-branes and moduli stabilization," hep-th/0311250. 